\title{
Tangent spaces of the Teichmüller space of the torus with Thurston's weak metric
}

\author{
Hideki Miyachi, Ken'ichi Ohshika and Athanase Papadopoulos
}

\begin{abstract}
In this paper, we show that the analogue of Thurston's asymmetric metric on the Teichmüller space of flat structures on the torus is weak Finsler and we give a geometric description of its unit circle at each point in the tangent space to Teichmüller space. We then introduce a family of weak Finsler metrics which interpolate between Thurston's asymmetric metric and the Teichmüller metric of the torus (which coincides with the hyperbolic metric). We describe the unit tangent circles of the metrics in this family.

\section{Thurstonin heikolla metriikalla varustetun rengaspinnan Teichmüllerin avaruuden tangenttiavaruudet}

Tiivistelmä. Tässä työssä osoitamme, että Thurstonin epäsymmetrisen metriikan vastine rengaspinnan laakeiden rakenteiden Teichmüllerin avaruudessa on heikko Finslerin metriikka, ja kuvailemme geometrisesti tämän metriikan yksikköympyrän Teichmüllerin avaruuden tangenttiavaruuden jokaisessa pisteessä. Sitten esittelemme heikkojen Finslerin metriikoiden perheen, joka interpoloi Thurstonin epäsymmetrisen metriikan ja (hyperbolisen metriikan kanssa yhtyvän) rengaspinnan Teichmüllerin metriikan välillä. Kuvailemme tämän perheen metriikoiden tangenttiavaruuden yksikköympyrät.
\end{abstract}

\section{Preliminaries}

We shall use the following identification between the Teichmüller space of the torus and the upper half-plane model of the hyperbolic plane $\mathbb{H}$ : Let $T^{2}$ be a twodimensional torus and fix a pair of generators $a, b$ of $\pi_{1}\left(T^{2}\right)$ represented by two simple closed curves on this surface intersecting at one point. The Teichmüller space of $T^{2}$, denoted by $\mathcal{T}\left(T^{2}\right)$, is the set of equivalence classes of pairs $(\Sigma, f)$, where $\Sigma$ is a Riemann surface and $f: T^{2} \rightarrow \Sigma$ an orientation-preserving homeomorphism, and where two pairs $\left(\Sigma_{1}, f_{1}\right),\left(\Sigma_{2}, f_{2}\right)$ are defined to be equivalent when $f_{1} \circ f_{2}^{-1}$ is isotopic to a biholomorphism. From the uniformisation theorem, for every point $x$ in $\mathcal{T}\left(T^{2}\right)$, there is a unique complex number $\zeta$ with $\operatorname{Im}(\zeta)>0$ such that $x$ is represented by the pair $(\mathbb{C} /(\mathbb{Z}+\zeta \mathbb{Z}), f)$, where $f$ is a homeomorphism taking the homotopy classes of $a, b$ to $1, \zeta \in \mathbb{Z}+\zeta \mathbb{Z}=\pi_{1}(\mathbb{C} /(\mathbb{Z}+\zeta \mathbb{Z}))$ respectively. In this way, $\mathcal{T}\left(T^{2}\right)$ is identified with $\mathbb{H}=\{z \in \mathbb{C} \mid \operatorname{Im}(z)>0\}$. This identification induces an isometry when the Teichmüller space $\mathcal{T}\left(T^{2}\right)$ is equipped with the Teichmüller metric and $\mathbb{H}$ is equipped with the metric of constant curvature -4 . In the sequel, we shall refer to this metric on $\mathbb{H}$ as the hyperbolic metric $d_{\text {hyp }}$. The isometry between the space $\mathcal{T}\left(T^{2}\right)$ equipped with the so-called Teichmüller metric and the space $\mathbb{H}$ equipped with the hyperbolic metric is a result of Teichmüller, see [4, Section 9] and [5, Section 9] for an English translation of Teichmüller's paper.

https://doi.org/10.54330/afm.113702

2020 Mathematics Subject Classification: Primary 53B40, 30F60, 32G15.

Key words: Thurston metric, Teichmüller space, Teichmüller metric, Finsler manifold.

This work is supported by JSPS KAKENHI Grant Numbers 16K05202, partially, 16H03933, $17 \mathrm{H} 02843$.

(c) 2022 The Finnish Mathematical Society 
We also need the following notion: A weak metric $\delta$ on a set $X$ is a map $\delta: X \times$ $X \rightarrow \mathbb{R}$ satisfying the following:

(1) $\delta(x, x)=0$ for every $x$ in $X$;

(2) $\delta(x, y) \geq 0$ for every $x$ and $y$ in $X$;

(3) $\delta(x, y)+\delta(y, z) \geq \delta(x, z)$ for every $x, y$ and $z$ in $X$.

In the paper [1], the following weak metric was introduced on $\mathbb{H}$ : First, for $\zeta_{1}, \zeta_{2} \in \mathbb{H}$, we let

$$
M\left(\zeta_{1}, \zeta_{2}\right)=\sup _{x \in \mathbb{R}}\left|\frac{\zeta_{2}-x}{\zeta_{1}-x}\right| .
$$

The weak metric $\delta$ is then defined by setting $\delta\left(\zeta_{1}, \zeta_{2}\right)=\log M\left(\zeta_{1}, \zeta_{2}\right)$.

In the same paper, the following explicit expression of $\delta$ was obtained:

$$
\delta\left(\zeta_{1}, \zeta_{2}\right)=\log \left(\frac{\left|\zeta_{2}-\bar{\zeta}_{1}\right|+\left|\zeta_{2}-\zeta_{1}\right|}{\left|\zeta_{1}-\bar{\zeta}_{1}\right|}\right)
$$

We note that this implies that

$$
\delta\left(\zeta_{2}, \zeta_{1}\right)=\delta\left(\zeta_{1}, \zeta_{2}\right)+\log \frac{\operatorname{Im}\left(\zeta_{1}\right)}{\operatorname{Im}\left(\zeta_{2}\right)} .
$$

It was also shown that this weak metric has the following two properties:

(1) The arithmetic symmetrisation of the weak metric $\delta$, that is, the weak metric $S \delta$ defined by

$$
S \delta\left(\zeta_{1}, \zeta_{2}\right)=\frac{1}{2}\left(\delta\left(\zeta_{1}, \zeta_{2}\right)+\delta\left(\zeta_{2}, \zeta_{1}\right)\right)
$$

is a genuine metric and coincides with the hyperbolic metric of the upper half-plane.

(2) The weak metric $\delta$ is an analogue for the torus of Thurston's asymmetric metric on Teichmüller space.

The last statement needs some explanation, and we give it now.

For any two points $z_{1}, z_{2}$ in the Teichmüller space $\mathcal{T}\left(T^{2}\right)$, we take representatives $\left(\Sigma_{1}=\mathbb{C} /\left(\mathbb{Z}+\zeta_{1} \mathbb{Z}\right), f_{1}\right),\left(\Sigma_{2}=\mathbb{C} /\left(\mathbb{Z}+\zeta_{2} \mathbb{Z}\right), f_{2}\right)$, and we regard them as tori equipped with the quotient flat metrics induced by the flat metric of the Euclidean plane. We set $\delta\left(z_{1}, z_{2}\right)=\delta\left(\zeta_{1}, \zeta_{2}\right)$. In [1], a weak metric on $\mathcal{T}\left(T^{2}\right)$ was defined as follows. Let $\mathcal{S}\left(T^{2}\right)$ denote the set of homotopy classes of essential simple closed curves on the torus. We set,

$$
\kappa\left(z_{1}, z_{2}\right)=\log \sup _{s \in \mathcal{S}\left(T^{2}\right)} \frac{\operatorname{length}_{\Sigma_{2}}\left(f_{2}(s)\right)}{\operatorname{length}_{\Sigma_{1}}\left(f_{1}(s)\right)},
$$

where length denotes the length of a closed geodesic in the corresponding homotopy class. The formula for $\kappa\left(z_{1}, z_{2}\right)$ is the analogue, in this Euclidean setting, of the formula for Thurston's metric in the hyperbolic setting given in $[6, \mathrm{p}$. 8]. Theorem 3 of [1] says the following:

$$
\kappa\left(z_{1}, z_{2}\right)=\delta\left(\zeta_{1}, \zeta_{2}\right)
$$

for any $z_{1}, z_{2} \in \mathcal{T}\left(T^{2}\right)$ and $z_{i}=\left(\Sigma_{i}=\mathbb{C} /\left(\mathbb{Z}+\zeta_{i} \mathbb{Z}\right), f_{i}\right)$ for $i=1,2$.

The metric $\delta$ has another characterisation which is given in [1]. For two metrics $g_{1}, g_{2}$ on $T_{0}=\mathbb{C} / \mathbb{Z} \oplus i \mathbb{Z}$ and a homeomorphism $\varphi: T_{0} \rightarrow T_{0}$, we define

$$
\mathcal{L}(\varphi)=\sup _{x \neq y}\left(\frac{d_{g_{2}}(\varphi(x), \varphi(y)) / \text { length }_{g_{2}}(s)}{d_{g_{1}}(x, y) / \text { length }_{g_{1}}(s)}\right),
$$


where $s$ is an essential simple closed curve, and set

$$
\lambda\left(g_{1}, g_{2}\right)=\inf _{\varphi}(\log \mathcal{L}(\varphi)),
$$

where $\varphi$ ranges over all homeomorphisms homotopic to the identity. The function $\lambda$ is invariant under the action of homeomorphisms on $T_{0}$ homotopic to the identity. Hence, $\lambda$ defines a weak metric on $\mathcal{T}\left(T^{2}\right)$. The metric is called the normalised weak Lipschitz distance. In [1], it was shown that $\kappa\left(z_{1}, z_{2}\right)=\lambda\left(g_{1}, g_{2}\right)$ for any flat metrics $g_{1}$ and $g_{2}$ representing $z_{1}$ and $z_{2}$ in $\mathcal{T}\left(T^{2}\right)$.

In the rest of this paper, we investigate further properties of the weak metric $\kappa=\delta$. We first show that the geodesics of the hyperbolic metric of $\mathbb{H}$ are geodesics with respect to this weak metric. We then show that this metric is weak Finsler (in a sense we shall make precise) and we give a geometric description of its unit circle at each point in the tangent space to Teichmüller space. We then introduce a family of weak Finsler metrics which interpolates between the weak metric $\delta$ and the hyperbolic metric (which coincides with the Teichmüller metric) which arises naturally from the construction given in this paper. We describe the unit tangent circle at each point for each weak metric in this family.

\section{Geodesics for the weak metric $\delta$}

In this section, we give an explicit expression for the point where the supremum of (1.1) is attained for given $\zeta_{1}, \zeta_{2} \in \mathbb{H}$ and show its geometric meaning. First we note the following, which can be shown easily from the definition of $\delta$ :

Lemma 2.1. For $\lambda>0$ and $\tau \in \mathbb{R}$, we have

$$
\begin{aligned}
\delta\left(\lambda \zeta_{1}+\tau, \lambda \zeta_{2}+\tau\right) & =\delta\left(\zeta_{1}, \zeta_{2}\right), \\
\delta\left(-\overline{\zeta_{1}},-\overline{\zeta_{2}}\right) & =\delta\left(\zeta_{1}, \zeta_{2}\right) .
\end{aligned}
$$

For $\zeta_{1}=a+i b$ and $\zeta_{2}=\alpha+i \beta$ in $\mathbb{H}$ with $\zeta_{1} \neq \zeta_{2}$, we define

$$
x_{ \pm}=\frac{\alpha^{2}+\beta^{2}-a^{2}-b^{2}}{2(\alpha-a)} \mp \frac{\sqrt{(\alpha-a)^{2}+(\beta-b)^{2}} \sqrt{(\alpha-a)^{2}+(\beta+b)^{2}}}{2(\alpha-a)}
$$

if $a \neq \alpha$. When $a=\alpha$, we define

$$
\begin{aligned}
& x_{+}= \begin{cases}0 & (\beta>b) \\
\infty & (\beta<b),\end{cases} \\
& x_{-}= \begin{cases}\infty & (\beta>b) \\
0 & (\beta<b) .\end{cases}
\end{aligned}
$$

The following is an explicit expression for the supremum in (1.1):

Proposition 2.1. For $\zeta_{1}, \zeta_{2} \in \mathbb{H}$ with $\zeta_{1} \neq \zeta_{2}$, the supremum in (1.1) is attained at $x_{+}$.

Proof. Let $\zeta_{1}=a+i b$ and $\zeta_{2}=\alpha+i \beta$. The case where $a=\alpha$ can be easily dealt with. The case where $\alpha<a$, from $(2.2)$, by considering $-\overline{\zeta_{1}}$ and $-\overline{\zeta_{2}}$ instead of $\zeta_{1}$ and $\zeta_{2}$ respectively, is reduced to the case where $a<\alpha$. Hence we only consider the case where $a<\alpha$.

We first assume that $a=0$. By assumption, we have $\alpha>0$. Set

$$
f(x)=\left|\frac{\zeta_{2}-x}{\zeta_{1}-x}\right|^{2}=\frac{(x-\alpha)^{2}+\beta^{2}}{x^{2}+b^{2}}=1+\frac{A+B x}{x^{2}+b^{2}}
$$


for $x \in \mathbb{R}$, where $A=\alpha^{2}+\beta^{2}-b^{2}$ and $B=-2 \alpha$. Then,

$$
f^{\prime}(x)=-\frac{B x^{2}+2 A x-B b^{2}}{\left(x^{2}+b^{2}\right)^{2}}
$$

and the critical points of $f^{\prime}(x)$ are

$$
x_{ \pm}=-\frac{A}{B} \pm \frac{\sqrt{A^{2}+b^{2} B^{2}}}{B} .
$$

Since $\operatorname{Re}\left(\zeta_{2}\right)=\alpha>0$ and $B=-2 \alpha<0$, we have $x_{+}<-A / B<x_{-}$. Therefore, $f(x)$ attains its maximum at

$$
\begin{aligned}
x_{+} & =-\frac{A}{B}+\frac{\sqrt{A^{2}+b^{2} B^{2}}}{B} \\
& =\frac{\alpha^{2}+\beta^{2}-b^{2}}{2 \alpha}-\frac{\sqrt{\alpha^{2}+(b-\beta)^{2}} \sqrt{\alpha^{2}+(b+\beta)^{2}}}{2 \alpha} .
\end{aligned}
$$

Suppose next that $a \neq 0$ and $\alpha>a$. From the invariance (2.1) and the above calculation, by considering $\zeta_{1}-a=i b$ and $\zeta_{2}-a=(\alpha-a)+i \beta$ instead of $\zeta_{1}$ and $\zeta_{2}$, we see that the maximum is attained at

$$
a+\frac{(\alpha-a)^{2}+\beta^{2}-b^{2}}{2(\alpha-a)}-\frac{\sqrt{(\alpha-a)^{2}+(b-\beta)^{2}} \sqrt{(\alpha-a)^{2}+(b+\beta)^{2}}}{2(\alpha-a)}
$$

which is equal to $x_{+}$in $(2.3)$.

Proposition 2.2. The points $x_{+}$and $x_{-}$in Proposition 2.1 are the endpoints at infinity of the hyperbolic geodesic line in $\mathbb{H}$ passing through $\zeta_{2}$ and $\zeta_{1}$. The point $x_{+}$lies on the side of $\zeta_{1}$, and $x_{-}$lies on the side of $\zeta_{2}$.

Proof. Set $\zeta_{1}=a+i b$ and $\zeta_{2}=\alpha+i \beta$ again. The case where $a=\alpha$ can be easily dealt with. Hence, as before, we may assume that $a=0$ and $\alpha>0$, and set $A=\alpha^{2}+\beta^{2}-b^{2}$ and $B=-2 \alpha$ as in the proof of Proposition 2.1. Then

$$
\begin{aligned}
\left|\zeta_{1}-\left(-\frac{A}{B}\right)\right|^{2} & =\left|i b-\left(-\frac{A}{B}\right)\right|^{2}=\frac{A^{2}}{B^{2}}+b^{2}=\frac{A^{2}+b^{2} B^{2}}{B^{2}}, \\
\left|\zeta_{2}-\left(-\frac{A}{B}\right)\right|^{2} & =\left|(\alpha+i \beta)-\left(-\frac{A}{B}\right)\right|^{2} \\
& =\left(\frac{A}{B}+\alpha\right)^{2}+\beta^{2}=\left(\frac{\alpha^{2}+\beta^{2}-b^{2}}{-2 \alpha}+\alpha\right)^{2}+\beta^{2} \\
& =\frac{\left(\alpha^{2}-\beta^{2}+b^{2}\right)^{2}+4 \alpha^{2} \beta^{2}}{4 \alpha^{2}} \\
& =\frac{\left(\alpha^{2}+(\beta-b)^{2}\right)\left(\alpha^{2}+(\beta+b)^{2}\right)}{4 \alpha^{2}}=\frac{A^{2}+b^{2} B^{2}}{B^{2}} .
\end{aligned}
$$

This means that $-A / B$ is the centre of the Euclidean semicircle perpendicular to the real axis passing through the points $\zeta_{1}$ and $\zeta_{2}$, and that $x_{+}, x_{-}$are the endpoints of this semicircle. Since $x_{+}<-A / B<x_{-}$, the four points $x_{+}, \zeta_{1}, \zeta_{2}$ and $x_{-}$lie on the semicircle in this order. Since such a semicircle is a hyperbolic geodesic, we have completed the proof.

Theorem 2.1. Hyperbolic geodesics in $\mathbb{H}$ are geodesic with respect to the weak metric $\delta$. Conversely, every geodesic with respect to $\delta$ is a hyperbolic geodesic. 
Proof. Suppose that $\zeta_{1}, \zeta_{2}$ and $\zeta_{3}$ lie on a hyperbolic geodesic $\gamma$ in this order. By Proposition 2.2, the endpoint at infinity $x$ of $\gamma$ which lies on the side of $\zeta_{1}$ not containing $\zeta_{2}, \zeta_{3}$ attains the supremum of (1.1) for $M\left(\zeta_{1}, \zeta_{2}\right), M\left(\zeta_{2}, \zeta_{3}\right)$ and $M\left(\zeta_{1}, \zeta_{3}\right)$, provided that $x \neq \infty$. Then by Eq. (1.1), we have

$$
M\left(\zeta_{1}, \zeta_{2}\right)=\frac{\left|\zeta_{2}-x\right|}{\left|\zeta_{1}-x\right|}, M\left(\zeta_{2}, \zeta_{3}\right)=\frac{\left|\zeta_{3}-x\right|}{\left|\zeta_{2}-x\right|}
$$

and

$$
M\left(\zeta_{1}, \zeta_{3}\right)=\frac{\left|\zeta_{3}-x\right|}{\left|\zeta_{1}-x\right|}
$$

This implies that $M\left(\zeta_{1}, \zeta_{3}\right)=M\left(\zeta_{1}, \zeta_{2}\right) M\left(\zeta_{2}, \zeta_{3}\right)$, hence $\delta\left(\zeta_{1}, \zeta_{3}\right)=\delta\left(\zeta_{1}, \zeta_{2}\right)+\delta\left(\zeta_{2}, \zeta_{3}\right)$. This means that $\gamma$ is a geodesic with respect to $\delta$.

If $x=\infty$, then in the same setting, we have $M\left(\zeta_{1}, \zeta_{2}\right)=M\left(\zeta_{2}, \zeta_{3}\right)=M\left(\zeta_{1}, \zeta_{3}\right)=$ 1 , and again $\gamma$ is a geodesic with respect to $\delta$.

Conversely, suppose that $\gamma$ is a geodesic with respect to $\delta$, and let $\zeta_{1}, \zeta_{2}, \zeta_{3}$ be arbitrary three points lying on $\gamma$ in this order. Then we have $\delta\left(\zeta_{1}, \zeta_{3}\right)=\delta\left(\zeta_{1}, \zeta_{2}\right)+\delta\left(\zeta_{2}, \zeta_{3}\right)$. By Eq. (1.3), this implies that $\delta\left(\zeta_{3}, \zeta_{1}\right)=\delta\left(\zeta_{3}, \zeta_{2}\right)+\delta\left(\zeta_{2}, \zeta_{1}\right)$, hence $S \delta\left(\zeta_{1}, \zeta_{3}\right)=$ $S \delta\left(\zeta_{1}, \zeta_{2}\right)+S \delta\left(\zeta_{2}, \zeta_{3}\right)$ for the arithmetic symmetrisation $S \delta$. Since $S \delta$ coincides with the hyperbolic metric, we see that $\gamma$ is also a hyperbolic geodesic.

Before discussing the connection with Teichmüller theory, we shall give a brief comment on this theory. The ideal boundary $\partial \mathbb{H}=\partial \mathcal{T}\left(T^{2}\right)$ is canonically identified with the Thurston compactification of $\mathcal{T}\left(T^{2}\right)$. Recall that the Thurston compactification of $\mathcal{T}\left(T^{2}\right)$ consists of the projective classes of measured foliations on the base surface (torus) $T_{0}=\mathbb{C} /(\mathbb{Z}+i \mathbb{Z})$. A measured foliation on $T_{0}$ is an equivalence class of a pair consisting of a foliation on $T_{0}$ together with a transverse measure. (Note that in the general Thurston theory, the foliations may have singular points, whereas in the case of the torus that we are discussing, the foliations are without singularities). Two such pairs are equivalent if they are isotopic. (In the general case, one has to include Whitehead moves in the equivalence relation, but in the case of the torus, there are no such moves.) For $\alpha \in \hat{\mathbb{R}}$, we define a measured foliation associated with $\alpha$ to be the pair consisting of the foliation obtained as integral curves of unit vectors satisfying $(1+|\alpha|)^{-1}(d x+\alpha d y)=0$ on $T_{0}$ and where the transverse measure is defined by $(1+|\alpha|)^{-1}|d x+\alpha d y|$. $\alpha$ is called the slope of the foliation. Notice that when $\alpha=\infty$, the associated measured foliation consists of the integral curves of the unit lines satisfying $d y=0$ equipped with the transverse measure $|d y|$.

The point $x_{+} \in \partial \mathbb{H}=\partial \mathcal{T}\left(T^{2}\right)$ discussed at the beginning of this section corresponds to the slope of the horizontal foliation of the Teichmüller map from $\zeta_{2}$ to $\zeta_{1}$. Geometrically, the leaves of the horizontal foliation are stretched under the deformation along the Teichmüller geodesic segment from $\zeta_{1}$ to $\zeta_{2}$ (see Figure 1).
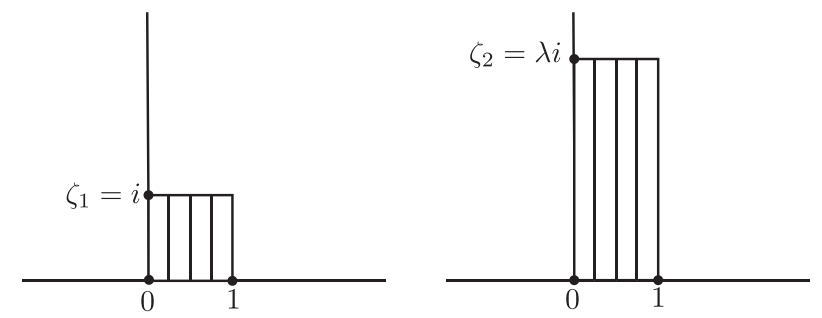

Figure 1. The Teichmüller ray (the hyperbolic geodesic ray) from $\zeta_{1}=i$ to $\zeta_{2}=\lambda i$ with $\lambda>1$ is the vertical ray emanating from from $\zeta_{1}$. In this case, $x_{+}=0$, and the leaves of the horizontal foliation are defined by $d x=0$, which is stretched along the deformation from $\zeta_{1}$ to $\zeta_{2}$. 
Combining Proposition 2.1 with Eq. (1.5), we have the following.

Corollary 2.1. Suppose that $z, z^{\prime} \in \mathcal{T}\left(T^{2}\right)$ correspond to $\zeta_{1}, \zeta_{2} \in \mathbb{H}$ respectively. Then the distance $\kappa\left(z, z^{\prime}\right)=\delta\left(\zeta_{1}, \zeta_{2}\right)$ is attained by the slope of the horizontal foliation for the Teichmüller map from $z$ to $z^{\prime}$.

\section{The weak Finsler structure of the weak metric $\delta$}

We recall now the notion of weak norm and weak Finsler metric on a manifold, adapted to the case we are dealing with. We start with a weak norm on a finitedimensional vector space $V$. This is a map $V \rightarrow[0, \infty), v \mapsto\|v\|$, satisfying

(1) $\|0\|=0$;

(2) $\|v\| \geq 0$ for all $v$ in $V$;

(3) $\left\|v+v^{\prime}\right\| \leq\|v\|+\left\|v^{\prime}\right\|$ for all $v$ and $v^{\prime}$ in $V$.

A metric on a smooth manifold $M$ is said to be weak Finsler if $M$ is equipped with a continuous field of weak norms defined on the tangent space at each point of $M$ such that the distance between two points in $M$ is equal to the infimum of the lengths of piecewise $C^{1}$-paths joining them, the length of such a path being computed as the integral over this path of the weak norms of the tangent vectors.

In this section, we show that the weak metric $\delta$ on $\mathcal{T}\left(T^{2}\right)$ is weak Finsler and we give a description of its induced weak norm on the tangent space of each point in this space. We start with the following proposition.

Proposition 3.1. Let $\zeta$ be a point in $\mathbb{H}$, and $v$ a tangent vector at $\zeta$. The weak metric $\delta$ induces on $v$ a weak norm $\|v\|_{\delta}$ expressed by

$$
\|v\|_{\delta}=\frac{|v|+\operatorname{Im}(v)}{2 \operatorname{Im}(\zeta)}
$$

The meaning of the expression "induced weak norm" will be clear from the computation done in the proof, and it acquires its complete significance in Corollary 3.1 which follows.

Proof. Set $\zeta^{\prime}=\zeta+t v(t>0)$. Then,

$$
\begin{aligned}
\left|\zeta^{\prime}-\zeta\right| & =|\zeta+t v-\zeta|=t|v| ; \\
\left|\zeta^{\prime}-\bar{\zeta}\right| & =|\zeta-\bar{\zeta}+t v|=|\zeta-\bar{\zeta}| 1+t \frac{v}{\zeta-\bar{\zeta}} \mid \\
& =|\zeta-\bar{\zeta}|\left(1+t \operatorname{Re}\left(\frac{v}{\zeta-\bar{\zeta}}\right)+o(t)\right) \\
& =|\zeta-\bar{\zeta}|\left(1+t \operatorname{Re}\left(\frac{v}{2 i \operatorname{Im}(\zeta)}\right)+o(t)\right) \\
& =2 \operatorname{Im}(\zeta)\left(1+t \frac{\operatorname{Im}(v)}{2 \operatorname{Im}(\zeta)}+o(t)\right) .
\end{aligned}
$$

Hence, we have

$$
\begin{aligned}
\delta(\zeta, \zeta+t v) & =\log \frac{1}{2 \operatorname{Im}(\zeta)}\left(t|v|+2 \operatorname{Im}(\zeta)\left(1+t \frac{\operatorname{Im}(v)}{2 \operatorname{Im}(\zeta)}+o(t)\right)\right) \\
& =\log \left(1+t \frac{|v|+\operatorname{Im}(v)}{2 \operatorname{Im}(\zeta)}+o(t)\right)=t \frac{|v|+\operatorname{Im}(v)}{2 \operatorname{Im}(\zeta)}+o(t)
\end{aligned}
$$


Thus, we obtain

$$
\lim _{t \searrow+0} \frac{\delta(\zeta, \zeta+t v)}{t}=\frac{|v|+\operatorname{Im}(v)}{2 \operatorname{Im}(\zeta)} .
$$

Notice that as an invariant expression, the weak metric in Proposition 3.1 is presented as

$$
\|\cdot\|_{\delta}=\frac{\sqrt{d x^{2}+d y^{2}}+d y}{2 y}=d s_{\text {hyp }}+\frac{1}{2} d \log y
$$

on $T_{\zeta} \mathbb{H}$ and $\zeta=x+i y \in \mathbb{H}$, where $d s_{\text {hyp }}$ is the length element of the hyperbolic metric on $\mathbb{H}$ of constant curvature -4 .

Consider now the hyperbolic space $\mathbb{H}$ equipped with the hyperbolic distance (of constant curvature -4 ) and let $\gamma$ be an isometrically parametrised geodesic emanating from a point $\zeta_{0}$ in $\mathbb{H}$ and converging to a point $x_{0} \in \partial \mathbb{H}$. We recall that the Busemann function associated with the geodesic ray $\gamma$ is defined by

$$
\mathbb{H} \ni \zeta \mapsto \lim _{t \rightarrow \infty}\left(d_{\text {hyp }}(\zeta, \gamma(t))-t\right)
$$

(cf. [3, Chapter 12]). Combining this with Theorem 2.1, we have the following corollary.

Corollary 3.1. The weak metric space $\left(\mathcal{T}\left(T^{2}\right), \delta\right)$ is a weak Finsler metric space with the corresponding weak norm $\|\cdot\|_{\delta}$ given in Proposition 3.1.

Proof. We first show that

$$
\int_{\theta_{1}}^{\theta_{2}}\|\dot{\gamma}(\theta)\|_{\delta} d \theta \geq \delta\left(\zeta_{1}, \zeta_{2}\right)
$$

for $\zeta_{1}, \zeta_{2} \in \mathbb{H}$ and any piecewise $C^{1}$-path $\gamma:\left[\theta_{1}, \theta_{2}\right] \rightarrow \mathbb{H}$ connecting $\zeta_{1}$ to $\zeta_{2}$. Indeed, from the invariant expression (3.1), the integration in the left-hand side of (3.2) is at least equal to the hyperbolic distance between $\zeta_{1}$ and $\zeta_{2}$ minus the difference of the Busemann functions at $\zeta_{1}, \zeta_{2}$ (see (4.2)), which is the right-hand side of (3.2) (cf. $\S 4)$. This observation also implies that the integration in the left-hand side of (3.2) is minimised only when it is done along the hyperbolic geodesic from $\zeta_{1}$ to $\zeta_{2}$.

We now show that the distance between any two points $\zeta_{1}$ and $\zeta_{2} \in \mathbb{H}$ is given by integrating the weak norm $\|\cdot\|_{\delta}$ along a parametrised geodesic joining these two points.

To this end, we first assume that $\operatorname{Re}\left(\zeta_{1}\right) \neq \operatorname{Re}\left(\zeta_{2}\right)$. As in the proof of Proposition 2.1, we may assume that $\zeta_{1}=i b$ and $\zeta_{2}=\alpha+i \beta$ with $\alpha>0$. Let $A=$ $\alpha^{2}+\beta^{2}-b^{2}, B=-2 \alpha$ and $R=\sqrt{A^{2}+b^{2} B^{2}} /|B|$. Define $\theta_{1}, \theta_{2} \in(0,2 \pi)$ by $e^{i \theta_{1}}=\left(\zeta_{1}-(-A / B)\right) / R$ and $e^{i \theta_{2}}=\left(\zeta_{2}-(-A / B)\right) / R$. Note that $\theta_{2}<\theta_{1}$. The geodesic from $\zeta_{1}$ to $\zeta_{2}$ is parametrised as $\gamma(\theta)=(-A / B)+R e^{i\left(\theta_{1}+\theta_{2}-\theta\right)}\left(\theta_{2} \leq \theta \leq \theta_{1}\right)$. Hence by setting $\phi$ to be $\theta_{1}+\theta_{2}-\theta$, we have

$$
\begin{aligned}
\int_{\theta_{2}}^{\theta_{1}}\|\dot{\gamma}(\theta)\|_{\delta} d \theta & =\int_{\theta_{2}}^{\theta_{1}} \frac{|\dot{\gamma}(\theta)|+\operatorname{Im}(\dot{\gamma}(\theta))}{2 \operatorname{Im}(\gamma(\theta))} d \theta=\int_{\theta_{2}}^{\theta_{1}} \frac{R-R \cos \left(\theta_{1}+\theta_{2}-\theta\right)}{2 R \sin \left(\theta_{1}+\theta_{2}-\theta\right)} d \theta \\
& =\int_{\theta_{2}}^{\theta_{1}} \frac{1-\cos (\phi)}{2 \sin (\phi)} d \phi=\frac{1}{2} \log \left(1+\cos \theta_{2}\right)-\frac{1}{2} \log \left(1+\cos \theta_{1}\right) \\
& =\frac{1}{2}\left(\log \left(1-\frac{2 \alpha^{2}-A}{\sqrt{A^{2}+b^{2} B^{2}}}\right)-\log \left(1+\frac{A}{\sqrt{A^{2}+b^{2} B^{2}}}\right)\right) .
\end{aligned}
$$


An easy calculation shows that this expression is equal to

$$
\log \frac{\sqrt{\alpha^{2}+(\beta-b)^{2}}+\sqrt{\alpha^{2}+(\beta+b)^{2}}}{2 b},
$$

that is, to $\delta\left(\zeta_{1}, \zeta_{2}\right)$. This gives what we wanted.

We now suppose that $\operatorname{Re}\left(\zeta_{1}\right)=\operatorname{Re}\left(\zeta_{2}\right)$. Then $\delta\left(\zeta_{1}, \zeta_{2}\right)$ is equal to zero when $\operatorname{Im}\left(\zeta_{2}\right)<\operatorname{Im}\left(\zeta_{1}\right)$ and to $\delta\left(\zeta_{1}, \zeta_{2}\right)=d_{\text {hyp }}\left(\zeta_{1}, \zeta_{2}\right)$ otherwise. From (3.1), $\|v\|_{\delta}$ is equal to zero when $\operatorname{Im}(v)<0$ and to $\|v\|_{\text {hyp }}:=\sqrt{d s_{\text {hyp }}^{2}(v, v)}$ otherwise. Hence, the integral of the $\delta$-norm along the hyperbolic geodesic connecting $\zeta_{1}$ to $\zeta_{2}$ coincides with the $\delta$-distance from $\zeta_{1}$ to $\zeta_{2}$.

Next we describe the unit circle in the tangent space with respect to the weak norm $\|\cdot\|_{\delta}$.

Proposition 3.2. The unit circle of the tangent space at $\zeta \in \mathbb{H}$ with respect to $\|\cdot\|_{\delta}$ is expressed as a parabola with focus at the origin and vertex at $i \operatorname{Im}(\zeta)$.

Proof. Let $\zeta=\alpha+i \beta$. When $v=v_{1}+i v_{2} \in \mathbb{C} \cong T_{\zeta} \mathbb{H}$ (as real vector spaces) lies on the unit circle of the tangent space at $\zeta$, we have

$$
1=\frac{|v|+\operatorname{Im}(v)}{2 \operatorname{Im}(\zeta)}=\frac{\sqrt{v_{1}^{2}+v_{2}^{2}}+v_{2}}{2 \beta}
$$

which is equivalent to

$$
v_{1}^{2}+v_{2}^{2}=\left(2 \beta-v_{2}\right)^{2}=4 \beta^{2}-4 \beta v_{2}+v_{2}^{2} .
$$

This means that the unit tangent circle at $\zeta=\alpha+i \beta \in \mathbb{H}$ is the parabola

$$
v_{2}=-\frac{v_{1}^{2}}{4 \beta}+\beta
$$

which implies the desired result.

Note that the fact that the unit tangent circle of the weak Finsler norm has an infinite direction expresses the fact that the distance function is degenerate in this direction (that is, we have, in this direction, $\delta(x, y)=0$ for $x \neq y$ ).

\section{Deforming $\delta$ to the Teichmüller metric}

In this section, we consider a family of weak Finsler metrics which interpolate between $\delta$ and the hyperbolic distance (which, as is well known, coincides with the Teichmüller distance). We then describe the unit tangent circle of each of these metrics.

Consider the family of weak metrics $\delta_{p}(0 \leq p \leq 1)$ defined by

$$
\delta_{p}\left(\zeta_{1}, \zeta_{2}\right)=\log \sup _{x \in \mathbb{R}} \frac{\left(\operatorname{Im}\left(\zeta_{1}\right)\right)^{p / 2}}{\left(\operatorname{Im}\left(\zeta_{2}\right)\right)^{p / 2}}\left|\frac{\zeta_{2}-x}{\zeta_{1}-x}\right|=\frac{p}{2} \log \frac{\operatorname{Im}\left(\zeta_{1}\right)}{\operatorname{Im}\left(\zeta_{2}\right)}+\log \sup _{x \in \mathbb{R}}\left|\frac{\zeta_{2}-x}{\zeta_{1}-x}\right| .
$$

Note that the function

$$
\mathbb{H} \ni \zeta \mapsto \frac{1}{2} \log \frac{\operatorname{Im}\left(\zeta_{0}\right)}{\operatorname{Im}(\zeta)}=-\frac{1}{2} \int_{\zeta_{0}}^{\zeta} d \log y
$$

is the Busemann function associated with the geodesic ray emanating from some fixed point $\zeta_{0} \in \mathbb{H}$ converging to $x=\infty \in \partial \mathbb{H}$ of the hyperbolic metric of curvature 
-4 , which is the Teichmüller distance. Hence, the function

$$
\frac{1}{2} \log \frac{\operatorname{Im}\left(\zeta_{1}\right)}{\operatorname{Im}\left(\zeta_{2}\right)}=\frac{1}{2} \log \frac{\operatorname{Im}\left(\zeta_{0}\right)}{\operatorname{Im}\left(\zeta_{2}\right)}-\frac{1}{2} \log \frac{\operatorname{Im}\left(\zeta_{0}\right)}{\operatorname{Im}\left(\zeta_{1}\right)}
$$

that appears in (4.1) is the difference of the Busemann functions. Using the same proof as in Theorem 2.1, the hyperbolic geodesic from $\zeta_{1}$ to $\zeta_{2}$ is the geodesic of the metric $\delta_{p}$. The arithmetic symmetrisation of $\delta_{p}$ is the hyperbolic metric of curvature -4 , like for $\delta=\delta_{0}$ (cf. (1) in $\S 1$ ).

As we did in Proposition 3.1, we can calculate the infinitesimal form of the metric $\delta_{p}$ : Let $\zeta \in \mathbb{H}$. For $v \in T_{\zeta} \mathbb{H} \cong \mathbb{C}$,

$$
\log \frac{\operatorname{Im}(\zeta)}{\operatorname{Im}(\zeta+t v)}=-\log \left(1+t \frac{\operatorname{Im}(v)}{\operatorname{Im}(\zeta)}\right)=-t \frac{\operatorname{Im}(v)}{\operatorname{Im}(\zeta)}+o(t)
$$

as $t \searrow+0$. We obtain

$$
\begin{aligned}
\|v\|_{\delta_{p}}:=\lim _{t \searrow+0} \frac{\delta_{p}(\zeta, \zeta+t v)}{t} & =-\frac{p}{2} \frac{\operatorname{Im}(v)}{\operatorname{Im}(\zeta)}+\frac{|v|+\operatorname{Im}(v)}{2 \operatorname{Im}(\zeta)} \\
& =\frac{|v|+(1-p) \operatorname{Im}(v)}{2 \operatorname{Im}(\zeta)}=\|v\|_{\delta}-p \frac{\operatorname{Im}(v)}{2 \operatorname{Im}(\zeta)}
\end{aligned}
$$

Notice that $\|v\|_{\delta_{p}}>0$ when $v \neq 0$ and $p>0$. The unit tangent circle with respect to the weak norm $\|\cdot\|_{\delta_{p}}$ in the tangent space $T_{\zeta} \mathbb{H}$ is the ellipse with foci 0 and $-4 \operatorname{Im}(\zeta)(1-p) /(p(2-p))$ (see Figure 2).

As an invariant expression, the weak metric $\|\cdot\|_{\delta_{p}}$ is presented as

$$
\|\cdot\|_{\delta_{p}}=\frac{\sqrt{d x^{2}+d y^{2}}+(1-p) d y}{2 y}=d s_{\text {hyp }}+\frac{1-p}{2} d \log y .
$$

A discussion similar to that of the proof of Corollary 3.1 and (4.3) shows that the weak metric space $\left(\mathcal{T}\left(T^{2}\right), \delta_{p}\right)$ is a weak Finsler metric with associated weak norm $\|\cdot\|_{\delta_{p}}$ and that the hyperbolic geodesic from $\zeta_{1}$ to $\zeta_{2} \in \mathbb{H}$ is a unique geodesic for $\delta_{p}$. Notice that $\|\cdot\|_{\delta_{1}}$ is the norm induced from the hyperbolic metric. From (4.3), $\|v\|_{\delta_{1}} \leq\|v\|_{\delta_{p}}^{2} \leq(2-p)\|v\|_{\delta_{1}}$ for $0<p \leq 1$. Hence $\delta_{p}$ and $\delta_{q}$ are bi-Lipschitzequivalent for $0<p, q \leq 1$. In particular, for $0<p \leq 1, \delta_{p}$ is complete and separates points in $\mathbb{H}$.

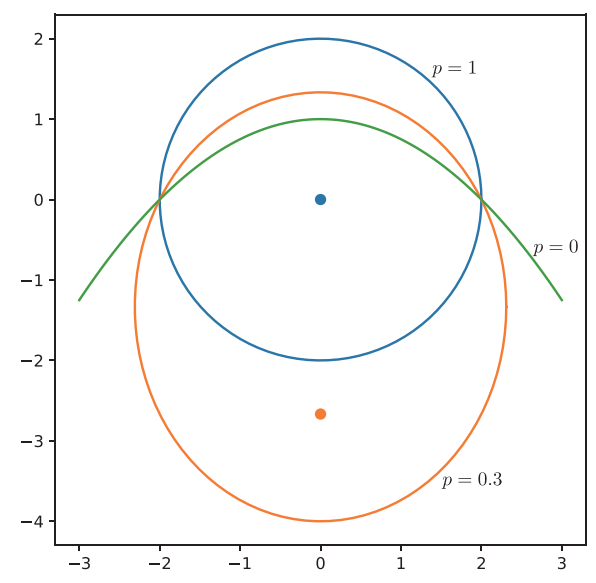

Figure 2. The infinitesimal unit circle of the weak norm $\|\cdot\|_{\delta_{p}}$ at $\zeta=0$ for $p=1,0.3$, and 0 . Each infinitesimal unit circle has the origin as a focus, and the lower dot is another focus for the infinitesimal unit circle for $p=0.3$. 
It follows that $\left\{\delta_{p}\right\}_{0 \leq p \leq 1}$ is a continuous family of weak Finsler metrics giving a deformation from $\delta=\delta_{0}$ to the hyperbolic metric $\delta_{1}$ (which is the Teichmüller metric). Notice that

$$
\frac{|\zeta-x|^{2}}{\operatorname{Im}(\zeta)}
$$

coincides with the extremal length of the measured foliation corresponding to $x \in \mathbb{R}$ up to a constant factor (depending only on $x$ ). (We recall that the extremal length of a simple closed curve $c$ on a Riemann surface is defined to be the infimum of the reciprocals of the moduli of the annuli whose core curves are homotopic to $c$, and the extremal length function can be extended continuously to the space of measured foliations.) Hence, our expression of $\delta_{1}$ in (4.1) coincides with Kerckhoff's formula for the Teichmüller distance [2] adapted to the case of the torus.

Acknowledgements. The authors would like to thank the referee for his careful reading and his insightful remarks and suggestions which improved the paper.

\section{References}

[1] Belkhirat, A., A. Papadopoulos, and M. Troyanov: Thurston's weak metric on the Teichmüller space of the torus. - Trans. Amer. Math. Soc. 357:8, 2005, 3311-3324.

[2] Kerckhoff, S. P.: The asymptotic geometry of Teichmüller space. Topology 19:1, 1980, 23-41.

[3] Papadopoulos, A.: Metric spaces, convexity and non-positive curvature. Second edition. IRMA Lect. Math. Theor. Phys. 6, Eur. Math. Soc., Zürich, 2014.

[4] Teichmüller, O.: Extremale quasikonforme Abbildungen und quadratische Differentiale. Abh. Preuss. Akad. Wiss. Math.-Nat. Kl. 1939:22, 1940, 197.

[5] Teichmüller, O.: Extremal quasiconformal mappings and quadratic differentials. -In: Handbook of Teichmüller theory, vol. V (edited by A. Papadopoulos), IRMA Lect. Math. Theor. Phys. 26, Eur. Math. Soc., Zürich, 2016, 321-483.

[6] Thurston, W. P.: Minimal stretch maps between hyperbolic surfaces. - arXiv:math/9801039, 1986.

Received 12 July 2020 • Accepted 18 June 2021 • Published online 21 January 2022

Hideki Miyachi

Kanazawa University

College of Science and Engineering

School of Mathematics and Physics

Kakuma-machi, Kanazawa

Ishikawa, 920-1192, Japan

miyachi@se.kanazawa-u.ac.jp

Athanase Papadopoulos

Université de Strasbourg et CNRS

Institut de Recherche Mathématique Avancée

7 rue René Descartes

67084 Strasbourg Cedex, France

papadop@math.unistra.fr
Ken'ichi Ohshika

Gakushuin University

Department of Mathematics

Mejiro, Toshima-ku, Tokyo, Japan

ohshika@math.gakushuin.ac.jp 